Article

\title{
Fractional Prabhakar Derivative in Diffusion Equation with Non-Static Stochastic Resetting
}

\author{
Maike A. F. dos Santos (1) \\ Instituto de Física, Universidade Federal do Rio Grande do Sul, Caixa Postal 15051, Porto Alegre CEP 91501-970, \\ Brazil; santosmaikeaf@gmail.com
}

Received: 25 January 2019; Accepted: 2 March 2019; Published: 6 March 2019

\begin{abstract}
In this work, we investigate a series of mathematical aspects for the fractional diffusion equation with stochastic resetting. The stochastic resetting process in Evans-Majumdar sense has several applications in science, with a particular emphasis on non-equilibrium physics and biological systems. We propose a version of the stochastic resetting theory for systems in which the reset point is in motion, so the walker does not return to the initial position as in the standard model, but returns to a point that moves in space. In addition, we investigate the proposed stochastic resetting model for diffusion with the fractional operator of Prabhakar. The derivative of Prabhakar consists of an integro-differential operator that has a Mittag-Leffler function with three parameters in the integration kernel, so it generalizes a series of fractional operators such as Riemann-Liouville-Caputo. We present how the generalized model of stochastic resetting for fractional diffusion implies a rich class of anomalous diffusive processes, i.e., $\left\langle(\Delta x)^{2}\right\rangle \propto t^{\alpha}$, which includes sub-super-hyper-diffusive regimes. In the sequence, we generalize these ideas to the fractional Fokker-Planck equation for quadratic potential $U(x)=a x^{2}+b x+c$. This work aims to present the generalized model of Evans-Majumdar's theory for stochastic resetting under a new perspective of non-static restart points.
\end{abstract}

Keywords: Fokker-Planck equation; anomalous diffusion; fractional calculus; stochastic resetting; exact solutions

\section{Introduction}

Presently, stochastic processes are a powerful tool in theories that maximize efficiency in search processes [1,2] that range from looking for obstacles to models that include the process of restarting [3-5]. In particular, restarting a search process can increase the chances of finding the target since the target may be in a known region. However, the first search strategy may not be successful. An example of a resetting process in biology is associated with a protein that walks on a strand of DNA. Since DNA is a very long molecule it can bend so that the protein that walks on it can make big jumps or even return to a specific position, which makes it easier for a protein to look for specific sites in the DNA biomolecule [6-9]. The diffusive processes with resting or renewal have in fact been intensely studied in recent years, and the main reason for this is in understanding and modelling biological systems. The idea of stochastic resetting not is new, but the formula proposed by Evans and Majumdar [10] in diffusion revived a series of discussions about how achievement is entailed in a series of sophisticated mathematical models to describe a diffusive process with restart. The idea of stochastic resetting came to be investigated in other formalisms such as stochastic processes associated with the exponent of Hurst [11], coagulation diffusion process [12], non-equilibrium steady states [13], RNA polymerase process [14] and others. However, these processes have gained great notoriety in the context of the diffusion equation. 
In this context, the Fokker-Planck equation (FPE) successfully describes the irregular motion of microscopic particles subjected to a force. The applicability of the FPE is undoubtedly of great relevance in statistical physics [15]. In recent decades, FPE has gained several generalized formulations, including nonlinear forms [16], fractal media [17], fractional [18,19], etc. In particular, in the last decade the fractional FPE (or fractional diffusion) has found a special prominence in the description of biological phenomena [20-23]. The main reason for the success of this formulation is associated with the complex effects that the fractional calculation introduces in diffusive processes, such as memory effect and non-locality [24-27].

The fractional approach in the diffusion process amplifies the concept of diffusion in several aspects, such as non-locality, Non-Gaussian distributions, memory effects, ergodicity breaking [26-32]. The main characteristic of usual diffusion (or Brownian diffusion) is a linear evolution of time to the mean square displacement (MSD), i.e., $\left\langle(\Delta x)^{2}\right\rangle \propto t$. Nevertheless, the class of phenomena which is not described by the usual diffusion is commonly known as anomalous diffusion (or fractional diffusion), and can be classified by power-law function,

$$
\left\langle(x-\langle x\rangle)^{2}\right\rangle \propto \mathcal{K}_{\alpha} t^{\alpha},
$$

in which $\langle(\cdots)\rangle=\int_{\mathcal{R}}(\cdots) f(x) d x\left(f(x)\right.$ is a distribution function), $\mathcal{K}_{\alpha}$ is general diffusion coefficient with fractional dimension $\left[\mathcal{K}_{\alpha}\right]=\mathrm{cm}^{2} /[\mathrm{t}]^{\alpha}$. If $0<\alpha<1$ the system is sub-diffusive, for $1<\alpha<2$ the super-diffusion occurs. In particular cases, for $\alpha=2$ the diffusion is ballistic and for $2<\alpha$ the hyper-diffusive process occurs. Equation (1) was first reported by Richardson in 1926, in the study of diffusion in turbulent flows [33]. Scher and Montroll (1975) also reported Equation (1) in a study about the transport of charge carriers in amorphous semiconductors [34]. Montroll's works reveal that the random walks are stochastic processes strongly connected with medium characteristics. The Scher-Montroll theory is known as the continuous-time random walk (CTRW), and revealed a natural connection between generalized random walker and diffusion equation in the presence of fractional-order derivatives. Thus, the term "fractional dynamics" was originated [28]. Today, fractional calculus is one of the most promising mathematical instruments for elegantly introducing the concept of non-locality [17]. Daily, fractional derivatives are applied in physics. Among other examples, there are chaotic systems [35], fractional FPE for non-singular kernels [29,36], the fractional Schrödinger equation [37], and viscoelasticity theory [38]. In addition, we want to emphasize that there is a class of derivatives that unifies the concept of tempered functions with the integral functions of Prabhakar. This union has brought new formulations for fractional diffusive models, since they render the Riemann-Liouville-Caputo fractional derivatives as individual cases.

In this work, we propose an extension of the stochastic resetting theory proposed by Evans and Majumdar [10]. Our extension contains two factors that bring great novelty: (i) we incorporate temperate fractional derivatives [39] into the model so that we can incorporate memory effects; and (ii) we consider restart points that move in space. In Section 2 we introduce the definitions of fractional derivatives and the concept of temperate derivatives. In Section 3, we write a brief introduction on the model proposed by Evans and Majumdar to then introduce our ideas. We began the investigation of the simplest (non-fractional) case to the most complex case associated with the temperate derivative of Prabhakar [40]. In Section 3.4, we perform an analysis of MSD for the analyzed systems, showing in which time regimes the dynamics of the walkers can present the phenomenon known as anomalous diffusion. In addition, in Section 4, we consider a fractional FPE to generalize the diffusive analysis to situations in which the system is in the presence of external forces, addressing the potential that has a quadratic form in space. In addition, we investigate how the theory can include multiple resetting points that move with constant velocities in space, and what are the implications of this for the diffusion of walkers over time. Finally, in the Section 5 we address the conclusions and final considerations. 


\section{Preliminary Concepts about Tempered Fractional Calculus}

We review some notions and concepts used throughout the paper.

Fractional calculus is one of the most robust instruments to approach complex systems. The study began after the discussion [41,42] between L'Hospital and Leibniz about the possibility of realizing a non-integer derivative with order $\frac{1}{2}$ under a $f(x)$ function, example

$$
\frac{d^{\frac{1}{2}}}{d x^{\frac{1}{2}}} x^{n}=\text { What is the result? }
$$

Presently there are several definitions and references that bring, in detail, mathematical and applicable aspects of fractional derivatives [43]. The best-known definitions for the fractional derivative are associated with formulations made by Riemann, Liouville, and Caputo.

Definition 1. For a continuous function $f: \mathbb{R}^{+} \rightarrow \mathbb{R}$. The fractional derivative of Caputo, to arbitrary order $\alpha \in[0,+\infty)$ is defined by

$$
{ }_{C} \mathcal{D}_{t}^{\alpha} f(t)=\frac{1}{\Gamma[n-\alpha]} \int_{0}^{t} \frac{1}{\left(t-t^{\prime}\right)^{1+\alpha-n}} \frac{d^{n}}{d t^{\prime n}} f\left(t^{\prime}\right) d t^{\prime}, \quad t \in \mathbb{R},
$$

in which $\Gamma[\ldots]$ is the Gamma function and $n-1<\alpha<n$. Considering $\alpha \in[0,1)$, the Laplace transform implies

$$
\mathcal{L}\left\{C^{\mathcal{D}}{ }_{t}^{\alpha} f(t)\right\}=s^{\alpha} \tilde{f}(s)-s^{\alpha-1} f(0),
$$

in which the Laplace transform is defined by $\int_{0}^{\infty} d t e^{-s t} f(t)=\widetilde{f}(s)$.

For $\alpha \rightarrow n$ Equation (3) retrieves the usual $n$-order derivative. For more details see [43]. In the same way that the fractional derivative was defined, a corresponding fractional integral can be defined [43].

The definition (3) answers the question (Equation (2)) that L'Hospital put to Leibniz [42], which for the case of a power-law function is given by

$$
\frac{d^{\frac{1}{2}}}{d t^{\frac{1}{2}}} t^{n}=\frac{\Gamma[n+1]}{\Gamma\left[n+\frac{1}{2}\right]} t^{n-\frac{1}{2}}
$$

in which $n>0$. To $\alpha \in[0,1)$ we obtain

$$
\frac{d^{\alpha}}{d t^{\alpha}} t^{n}=\frac{\Gamma[n+1]}{\Gamma[n+1-\alpha]} t^{n-\alpha}
$$

Therefore, the fractional derivative for power laws has a mathematical structure similar to that of the integer-order derivative in Leibniz-Newton sense, i.e.,

$$
\lim _{\alpha \rightarrow 1} \frac{d^{\alpha}}{d t^{\alpha}} t^{n}=n t^{n-1}
$$

There are several other definitions of fractional derivatives, and these satisfy several mathematical properties that are detailed in [44]. These derivatives applied in differential equations generate a series of special functions [43], the Mittag-Leffler and Fox functions when applied in contexts associated with particle diffusion. In fact, the versatility of the fractional index $\alpha$ introduces the concept of memory if the derivative is applied to a temporal variable; an example occurs in CTRW [28], which brings a new vision to approach dynamic in disordered mediums, trap systems, and others [45]. On the other hand, 
the fractional derivative can be applied to the spatial variable, which implies non-locality; an example of this appears in the fractional Schrödinger equation [46].

The fractional derivatives, such as Riemann-Liouville, Letnikov, Riesz, and others, are constituted by convolution integrals with power-law kernels. The concept of tempered derivative appears when these kernels are multiplied by the exponential function [39,47-49] that corresponds to a truncated kernel. These fractional derivatives generalize the random walk problem and imply a larger class of non-Gaussian distributions.

Recently, the fractional Prabhakar derivative [40] has been the most complete and sophisticated tool for describing complexity in physical systems. The tempered Prabhakar derivative in Caputo sense is defined as follows

$$
\mathcal{D}_{\alpha, \beta, t}^{\delta, v, a} f(t)=\int_{0}^{t} e^{-a\left(t-t^{\prime}\right)} \wp_{\alpha, \beta}^{\delta, v}\left(t-t^{\prime}\right) \frac{d}{d t^{\prime}} f\left(t^{\prime}\right) d t^{\prime}, \quad t \in \mathbb{R},
$$

with the Prabhakar kernel $\wp_{\alpha, \beta}^{\delta, v}$ defined by

$$
\wp_{\alpha, \beta}^{\delta, v}[t]=t^{\beta-1} E_{\alpha, \beta}^{\delta}\left(-v t^{\alpha}\right),
$$

in which $E_{\alpha, \beta}^{\delta}(z)$ is the generalized Mittag-Leffler function for three parameters, given by [50]

$$
E_{\alpha, \beta}^{\delta}(z)=\sum_{k=0}^{\infty} \frac{(\delta)_{k}}{\Gamma[\alpha k+\beta]} \frac{z^{k}}{k !}
$$

$(\delta)_{k}=\Gamma[\delta+k] / \Gamma[\delta]$ is the Pochhammer symbol, with $\mathcal{R}\{\beta\}>0$ and $\beta, \alpha, \delta, z \in \mathbb{C}$. The function (10) recoveries the two-parameter Mittag-Leffler function [43] to $\delta=1$, i.e., $E_{\alpha, \beta}^{1}(z)$. The Mittag Leffler function (10) is reduced from one parameter to $\beta=1$ and $\delta=1$. Finally, the function (10) assumes the exponential form when $\alpha=\beta=\delta=1$, i.e., $E_{1,1}^{1}(z)=e^{z}$. Among the advantages of using the Prabhakar derivative (Equation (8)), there is the fact that the Caputo derivative ( or Riemann-Liouville, depending on how the Prabhakar derivative is defined) is a particular case of the Prabhakar derivative. The Laplace transform of Equation (9) is summarized as follows

$$
\mathcal{L}\left\{t^{\beta-1} E_{\alpha, \beta}^{\delta}\left(-v t^{\alpha}\right)\right\}=\frac{s^{\alpha \delta-\beta}}{\left(s^{\alpha}+v\right)^{\delta}} \quad \mathcal{R}\{s\}<|v|^{\frac{1}{\alpha}},
$$

in which $\delta, \alpha, \beta \in \mathbb{C}$ and $\mathcal{R}\{\alpha\}, \mathcal{R}\{\beta\}>0$. Equation (11) satisfies a series of mathematical proprieties that were detailed in [51]. Please note that for $\delta=0, a=0$ and $\beta=1-\alpha$, Equation (8) retrieves the fractional derivative form of Caputo; see Equation (3). The Prabhakar fractional derivative has revealed a class of interesting behaviors in the context of the viscoelasticity theory [52] and in anomalous advection-dispersion transport [53]. On the other hand, the fractional Prabhakar derivative has been an efficient tool in physical models to approach the transition among anomalous diffusions [54,55]. In particular, the first time that tempered Prabhakar derivative appears in diffusion context was in articles [56,57]. In fact, in this scenario, the Prabhakar derivative has been a mathematical tool that has been more understood day by day $[38,58,59]$. On the other hand, the Prabhakar derivative has as a particular case the Mittag-Leffler kernels with one and two parameters that have many applications in mathematical, physics, chemistry, and biology problems [35,36,60-62].

\section{Non-Static Stochastic Resetting Theory}

The stochastic resetting theory in the context of Brownian motion was introduced by Evans and Majumdar in 2011. They proposed a simple equation [10] as follows

$$
\frac{\partial}{\partial t} p(x, t)=\mathcal{K} \frac{\partial^{2}}{\partial x^{2}} p(x, t)-\kappa p(x, t)+\kappa \delta(x)
$$


with the following conditions

$$
p( \pm \infty, t)=0 \quad p(x, 0)=\delta(x)
$$

in which walkers are described by the probability distribution $p(x, t)$ and eventually restart at a specific point in the system. The stochastic resetting proposed by Evans-Majumdar (Equation (12)) is constituted by diffusion equation with additional terms. The second and the third terms on the right side of Equation (12) represent a sink function $-\kappa p(x, t)$ out of each point $x$ and a corresponding positive probability flux $\kappa \delta(x)$ which represents the reset position [10]. The reestablishment condition may be otherwise, or even associated with a previously specified probability distribution [10]. The theory proposed by Evans and Majumdar (12) has a certain mathematical similarity with the linear irreversible-reaction-diffusion equation, except in the stochastic resetting theory the walkers are never permanently removed from the system, i.e., $\int_{-\infty}^{+\infty} p(x, t) d x=1$. A consequence of this is that Equation (12) has a stationary distribution given by

$$
p_{s t}(x)=\frac{e^{-\sqrt{\frac{\kappa}{\mathcal{K}}}|x|}}{\mathcal{Z}}
$$

$\mathcal{Z}=2 \sqrt{\frac{\mathcal{K}}{\kappa}}$, which occurs due to a delicate balance between the walkers removed and those that are added in the initial position. In recent research, the theory of stochastic resetting in the Evans-Majumdar sense had several implications in statistical theories applied in physical systems ranging from Brownian motion approached through stochastic noises [63], diffusion with resetting and memory [64], search strategy optimization [65], first-passage time problem [66], telegraphic processes with stochastic resetting [67], diffusion with non-singular memory kernels [29], and others [68-70].

Our generalization consists of the combination of the fractional diffusion $[28,54]$ and stochastic resetting with memory [13]. To do this we consider the CTRW theory with an additional term $g(x, t)$ proposed by Henry and Wearne in [71]. This permits the removal or injection of walkers in the system. In this context, we have

$$
p(x, t)=\phi(t) p(x, 0)+\int_{0}^{t} \int_{-\infty}^{\infty} d t^{\prime} d x^{\prime} \Psi\left(x-x^{\prime}, t-t^{\prime}\right) p\left(x^{\prime}, t^{\prime}\right)+\int_{0}^{t} d t^{\prime} \phi\left(t-t^{\prime}\right) g\left(x, t^{\prime}\right) .
$$

In particular, $\Psi(x, t)=\lambda(x) \psi(t)$ represents the transition probability function, in which $\lambda(x)$ is the step length distribution function and $\psi(t)$ is the waiting time distribution. Now, we can define the cumulative probability as $\phi(t)=1-\int_{0}^{t} \psi\left(t^{\prime}\right) d t^{\prime}$ [28]. Therefore, we can rewrite Equation (15) in the Laplace space and Fourier space $\left(\bar{p}(k, t)=\mathcal{F}\{p(x, t)\}=\int_{-\infty}^{+\infty} p(x, t) e^{-i x k} d x\right)$ as follow

$$
\widetilde{\bar{p}}(k, s)=\widetilde{\phi}(s) \bar{p}(k, 0)+\widetilde{\Psi}(k, s) \widetilde{\bar{p}}(k, s)+\widetilde{\phi}(s) \widetilde{\bar{g}}(k, s),
$$

as $\widetilde{\phi}(s)=\frac{1}{s}(1-\widetilde{\psi}(s))$ and $\widetilde{\Psi}(k, s)=\bar{\lambda}(k) \widetilde{\psi}(s)$, we obtain

$$
s \tilde{\bar{p}}(k, s)=(1-\widetilde{\psi}(s)) \bar{p}(k, 0)+s \bar{\lambda}(k) \widetilde{\psi}(s) \widetilde{\bar{p}}(k, s)+(1-\widetilde{\psi}(s)) \widetilde{\bar{g}}(k, s) .
$$

The distribution of jump lengths with variance $\sigma^{2}$ can be approximated for $\bar{\lambda}(k) \sim 1-\frac{\sigma^{2}}{2} k^{2}$ [28]. Here, we can consider a waiting time distribution which describes a more general class of random walkers

$$
\psi(s)=\frac{1}{1+\widetilde{\wp}_{\alpha, \beta}^{\delta, v}[s+\kappa] \tau s},
$$


in which $\widetilde{\wp}_{\alpha, \mu}^{S, \nu}[s+\kappa]=\frac{(s+\kappa)^{\alpha \zeta-\mu}}{\left(v+(s+\kappa)^{\alpha}\right)^{\zeta}}$. The Equation (18) was reported and analyzed in [54]. Using Equation (18) in Equation (17), we obtain

$$
s \widetilde{\bar{p}}(k, s)=\frac{\widetilde{\wp}_{\alpha, \mu}^{S, v}[s+\kappa] \tau s}{1+\widetilde{\wp}_{\alpha, \mu}^{S, v}[s+\kappa] \tau s}(\bar{p}(k, 0)+\widetilde{\bar{g}}(k, s))+\frac{s}{1+\widetilde{\wp}_{\alpha, \mu}^{S, v}[s+\kappa] \tau s}\left(1-\frac{\sigma^{2}}{2} k^{2}\right) \widetilde{\bar{p}}(k, s),
$$

this equation can be rewritten as

$$
\tau s \widetilde{\wp}_{\alpha, \mu}^{s, v}[s+\kappa] \widetilde{\bar{p}}(k, s)=\tau \widetilde{\wp}_{\alpha, \mu}^{s, v}[s+\kappa](\bar{p}(k, 0)+\widetilde{\bar{g}}(k, s))-\frac{\sigma^{2}}{2} k^{2} \widetilde{\bar{p}}(k, s),
$$

thereby

$$
s \widetilde{\wp}_{\alpha, \mu}^{S, v}[s+\kappa] \widetilde{\bar{p}}(k, s)-\widetilde{\wp}_{\alpha, \mu}^{S, v}[s+\kappa] \bar{p}(k, 0)=-\mathcal{K} k^{2} \widetilde{\bar{p}}(k, s)+\widetilde{\wp}_{\alpha, \mu}^{S, v}[s+\kappa] \widetilde{\bar{g}}(k, s),
$$

in which $\mathcal{K}=\frac{\sigma^{2}}{2 \tau}$. For the stochastic resetting problem, we have $\widetilde{\bar{g}}=-\kappa \tilde{\bar{p}}(k, s)+\frac{\kappa}{s+i v k}$. Performing the inverse Fourier-Laplace transform of Equation (21), we obtain the fractional Prabhakar diffusion with non-static stochastic resetting, as follows

$$
\begin{aligned}
\mathcal{D}_{\alpha, \mu, t}^{\zeta, v, k} p(x, t) & =\mathcal{K} \frac{\partial^{2}}{\partial x^{2}} p(x, t) \\
& -\int_{0}^{t} d t^{\prime} e^{-\kappa\left(t-t^{\prime}\right)} \wp_{\alpha, \mu}^{\varsigma, \nu}\left[t-t^{\prime}\right]\left(\kappa p\left(x, t^{\prime}\right)-\kappa \delta\left(x-v t^{\prime}\right)\right),
\end{aligned}
$$

in which $v$ ís the propagation velocity of the resetting point and $\mathcal{D}_{\alpha, \mu, t}^{\zeta, v, k}$-operator was defined in Equation (8). This proposal considers two important factors in the diffusion process: tempered fractional derivative and non-static resetting term $\left((\cdots) \times \delta\left(x-v t^{\prime}\right)\right)$. The latter is exclusively due to the fact that the place where the particle restarts changes position in time. The principle for this change in the resetting position $x_{\text {reset }}(t)=v t$ is that if we imagine the problem of DNA, it is biologically more likely to assume a variable resetting position, because a protein walks over a long filament at different positions because the DNA molecule is too complex and there may be significant alterations in the resetting positions. On the other hand, we chose to investigate the fractional Prabhakar derivative in diffusion with stochastic resetting because of their generality, but here we can emphasize two reasons: (i) to solve the equation with fractional Prabhakar derivative corresponding to solving the usual derivative (Leibniz-Newton), fractional Caputo derivative [43] and Atangana-Baleanu operator [60]. All these operators have many applications in physics systems, e.g., ergodicity [32,72,73], strange kinetics of single molecules in living cells [74], relaxation in filled polymers [75], chaos in a simple nonlinear system [35], etc.; and (ii) the fractional Prabhakar derivative is one of the most sophisticated mathematical tools to approach the anomalous diffusion phenomena. However, is extremely difficult of solve a problem with Prabhakar derivative [76,77].

Equation (22) has many situations that are relevant. In [54] the authors derived the tempered Prabhakar diffusion equation making use of CTRW theory. For simplicity, in this section, we will consider that the system is governed by an equation that is free of external forces. First, we find the general solution in the Laplace-Fourier space, then make a directed analysis for each particular case. Later, in this section, we include a discussion of how quadratic potentials influence the dynamics of the system.

Applying Equation (22), we operate the Fourier transform in the spatial variable and Laplace in the time variable, and we obtain as a solution in the following general form

$$
\widetilde{\bar{p}}(k, s)=\frac{(\kappa+s)^{\alpha \varsigma-\mu}}{\left(v+(\kappa+s)^{\alpha}\right)^{\varsigma}} \frac{1+\kappa \frac{1}{s+i k v}}{\frac{(\kappa+s)^{1+\alpha \varsigma-\mu}}{\left(v+(\kappa+s)^{\alpha}\right)^{\varsigma}}+\mathcal{K} k^{2}},
$$


note that $\widetilde{\bar{p}}(k=0, s)=s^{-1}$, so $\int_{-\infty}^{+\infty} \widetilde{p}(x, s)=s^{-1}$. In other words, the number of walkers is conserved. From Equation (23) we calculate the solution for three cases: usual, fractional Caputo, and fractional Prabhakar.

\subsection{Non-Static Stochastic Resetting: Diffusion Equation}

In this subsection, we present a non-fractional case to the non-static stochastic resetting model. Here, we investigate the effect of the non-static restart point in diffusion.

The first case considers the usual diffusion, i.e., $\varsigma=\mu=0$. It corresponds to Equation (11) when the Prabhakar kernel tries the delta function, i.e., $\lim _{\zeta \rightarrow 0} \lim _{\mu \rightarrow 0} \wp_{\alpha, \mu}^{\zeta, v}(t)=\delta(t)$. The purpose here is to consolidate the simpler model, and to understand the effect of resetting that is non-static in Equation (22). In this case, $(\varsigma=\mu=0)$, the solution decreases as follows

$$
\widetilde{\bar{p}}(k, s)=\frac{1+\kappa \frac{1}{s+i k v}}{s+\mathcal{K} k^{2}+\kappa} .
$$

Realizing the inverse Laplace transform we obtain

$$
\widetilde{\bar{p}}(k, t)=e^{-t\left(\mathcal{K} k^{2}+\kappa\right)}+\kappa \int_{0}^{t} d t^{\prime} e^{-t^{\prime}\left(\mathcal{K} k^{2}+\kappa\right)-i k v\left(t-t^{\prime}\right)}
$$

now, realizing the inverse Fourier transform we obtain the exact solution:

$$
p(x, t)=\frac{e^{-\frac{x^{2}}{4 \mathcal{K} t}-\kappa t}}{2 \sqrt{\pi t \mathcal{K}}}+\kappa \int_{0}^{t} d t^{\prime} \frac{e^{-\frac{\left(x-v\left(t-t^{\prime}\right)\right)^{2}}{4 \mathcal{K} t^{\prime}}-t^{\prime} \kappa}}{2 \sqrt{\pi t^{\prime} \mathcal{K}}},
$$

This solution generates a series of interesting behaviors that is illustrated in Figure 1. On the other hand, for $v=0$ we retrieve the usual Evans-Majumdar model, changing the notation $t^{\prime} \rightarrow \tau$, if furthermore large time values, i.e., $t \rightarrow+\infty$, we obtain

$$
p_{s t}(x)=\int_{0}^{\infty} d \tau k e^{-k \tau} \frac{e^{-\frac{x^{2}}{4 \mathcal{K}}}}{2 \sqrt{\tau \pi \mathcal{K}}}
$$

realizing the integration we obtain Equation (14). The Equation (27) is a typical equation of renewal process [10]. In fact, if we consider that $\tau$ defines the time elapsed since the last renewal, such that $0 \leq \tau \leq t$ with $t \rightarrow \infty$. The part $\operatorname{Prob}(\tau)=\kappa e^{-\kappa \tau}$ defines how the renewal time $(\tau)$ is distributed, and $e^{-\frac{x^{2}}{4 \mathcal{K} \tau}} / 2 \sqrt{\tau \pi \mathcal{K}}$ is the Gaussian form that defines the position distribution. Thus, we prove that for the case $v=0$ we have the renewal process.

\subsection{Non-Static Stochastic Resetting: Fractional Caputo Diffusion Equation}

This case represents a fundamental limit to approach the fractional derivative of Caputo in the problem $(\varsigma=0)$. Therefore, Equation (23) can be written in the Fourier-Laplace space, as

$$
\widetilde{\bar{p}}(k, s)=(s+\kappa)^{-\mu} \frac{1+\kappa \frac{1}{s+i k v}}{(s+\kappa)^{1-\mu}+\mathcal{K} k^{2}} .
$$

Performing the inverse Fourier transform, we have

$$
p(x, s)=\frac{e^{-\sqrt{\frac{(s+\kappa)^{1-\mu}}{\mathcal{K}}}|x|}}{2 \sqrt{\mathcal{K}}(s+\kappa)^{\frac{1+\mu}{2}}}+\int_{-\infty}^{+\infty} d x^{\prime} \frac{e^{-\sqrt{\frac{(s+\kappa)^{1-\mu}}{K}}\left|x^{\prime}\right|}}{2 \sqrt{\mathcal{K}}(s+\kappa)^{\frac{1+\mu}{2}}} \chi\left(x-x^{\prime}, s\right)
$$


in which $\chi(x, s)=\mathcal{F}^{-1}\left\{\frac{1}{s+i k v}\right\}$, to perform the inverse Laplace transform we will introduce the following form [78]

$$
\mathcal{L}^{-1}\left\{s^{-\sigma} \exp \left[-z s^{\gamma}\right]\right\}=t^{\sigma-1} \mathrm{H}_{1,1}^{1,0}\left[\left.\frac{|z|}{t \gamma}\right|_{(0,1)} ^{(\sigma, \gamma)}\right]
$$

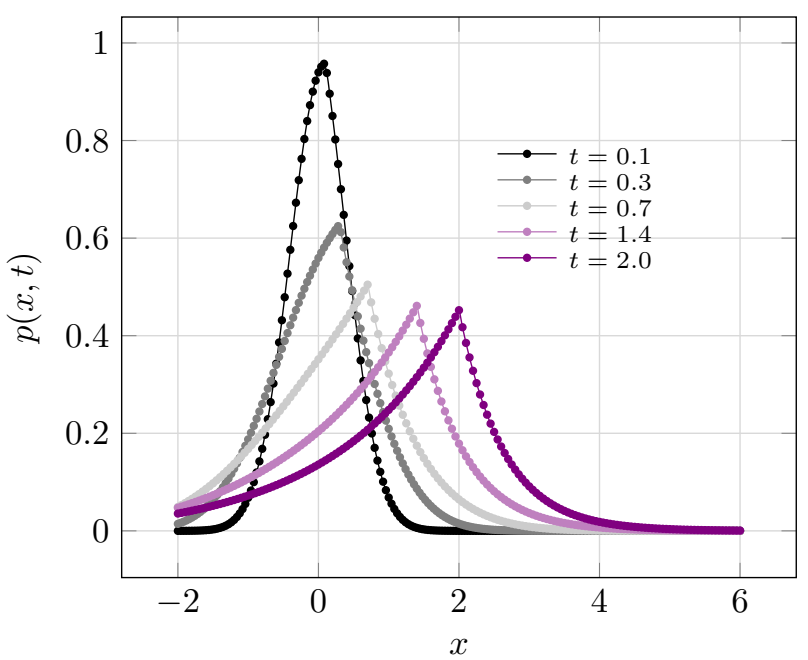

Figure 1. This figure exemplifies the evolution of probability distribution on time for the system when the velocity of the resetting points is given by $v=1$, with the following values $\kappa=1$ and $\mathcal{K}=1$.

The Fox $\mathrm{H}$ function (or H-function) may be defined in terms of the Mellin-Barnes type integral

$$
\mathrm{H}_{p, q}^{m, n}\left[\left.x\right|_{\left(b_{q}, B_{q}\right)} ^{\left(a_{p}, A_{p}\right)}\right]=\frac{1}{2 \pi i} \int_{L} \chi(\xi) x^{-\xi} d \xi
$$

with

$$
\chi(\xi)=\frac{\prod_{j=1}^{m} \Gamma\left[b_{j}-B_{j} \xi\right] \prod_{j=1}^{n} \Gamma\left[1-a_{j}+A_{j} \xi\right]}{\prod_{j=m+1}^{q} \Gamma\left[1-b_{j}+B_{j} \xi\right] \prod_{j=n+1}^{p} \Gamma\left[a_{j}-A_{j} \xi\right]}
$$

where $m, n, p$ and $q$ are integers satisfying $0 \leq n \leq p$ and $1 \leq m \leq q$. It may also be defined by its Mellin transform

$$
\int_{0}^{\infty} \mathrm{H}_{p, q}^{m, n}\left[\left.a x\right|_{\left(b_{q}, B_{q}\right)} ^{\left(a_{p}, A_{p}\right)}\right] x^{\xi-1} d x=a^{-\xi} \chi(\xi)
$$

Here, the parameters must be defined such that $A_{j}>0$ and $B_{j}>0$ and $a_{j}\left(b_{h}+v\right) \neq B_{h}\left(a_{j}-\lambda-1\right)$ where $v, \lambda=0,1,2, \ldots, h=1,2, \ldots, m$ and $j=1,2, \ldots, m$. The contour $L$ separates the poles of $\Gamma\left(b_{j}-B_{j} \xi\right)$ for $j=1,2, \ldots, m$ from those of $\Gamma\left(1-a_{j}+A_{j} \xi\right)$ for $j=1,2, \ldots, n$. The H-function is analytic in $x$ if either $(i) x \neq 0$ and $M>0$ or $(i i) 0<|x|<1 / B$ and $M=0$, where $M=\sum_{j=1}^{q} B_{j}-\sum_{j=1}^{p} A_{j}$ and $B=\prod_{j=1}^{p} A_{j}^{A_{j}} \prod_{j=1}^{q} B_{j}^{-B_{j}}$.

A closed-form solution for expression (28) can be found in terms of Fox functions 


$$
\begin{aligned}
p(x, t) & =e^{-\kappa t} \frac{t^{\frac{\mu-1}{2}}}{2 \sqrt{\mathcal{K}}} \mathrm{H}_{1,1}^{1,0}\left[\left.\frac{|x|}{\sqrt{\mathcal{K}} t^{\frac{1-\mu}{2}}}\right|_{(0,1)} ^{\left(\mu, \frac{1-\mu}{2}\right)}\right] \\
& +\int_{0}^{t} d t^{\prime} e^{-\kappa t^{\prime}} \frac{t^{\prime \frac{\mu-1}{2}}}{2 \sqrt{\mathcal{K}}} \mathrm{H}_{1,1}^{1,0}\left[\left.\frac{\left|x-v\left(t-t^{\prime}\right)\right|}{\sqrt{\mathcal{K}} t^{\prime \frac{1-\mu}{2}}}\right|_{(0,1)} ^{\left(\frac{\mu+1}{2}, \frac{1-\mu}{2}\right)}\right] .
\end{aligned}
$$

This expression retrieves Equation (37) to $\mu \rightarrow 0$.

As is the usual result obtained by us, we can assume a static stochastic resetting point, i.e., $v=0$ and the limit $t \rightarrow \infty$; the consequence of this is an expression that can be obtained by the renewal process, the unique change in relation to Equation (27) is the substitution of the Gaussian function by the solution of the fractional diffusion equation [19]. This particularity is interesting because it connects the fractional stochastic resetting model with a renewal process governed by generalized distributions. These results show that the proposed model Equation (22) connects the renewal process to stochastic resetting process such that $v=0$.

\subsection{Non-Static Stochastic Resetting: Fractional Prabhakar Diffusion Equation}

Finally, we will present the solution for Prabhakar diffusion. Solving the Prabhakar diffusion equation is a matter of complex diffusion. The simplest case in our model $(\kappa=0)$ had the solution found only in the Fourier space [51]. However, there are some techniques that we will present and may enable us to see the complete solution taking into consideration non-stochastic resetting. To do this, we can invert the Fourier transform in the general solution (Equation (23)); we obtain

$$
\begin{array}{r}
\widetilde{\bar{p}}(k, s)=\frac{(\kappa+s)^{-\mu}}{\left(v(\kappa+s)^{-\alpha}+1\right)^{s}} \frac{1+\kappa \frac{1}{s+i k v}}{\frac{(\kappa+s)^{1-\mu}}{\left(v(\kappa+s)^{-\alpha}+1\right)^{s}}+\mathcal{K} k^{2}}, \\
=\frac{1}{\kappa+s} \frac{1+\kappa \frac{1}{s+i k v}}{1+\frac{\left(\nu(\kappa+s)^{-\alpha}+1\right)^{s} \mathcal{K} k^{2}}{(\kappa+s)^{1-\mu}}}
\end{array}
$$

which can be rewritten as

$$
\begin{aligned}
\widetilde{p}(x, s) & =\mathcal{F}^{-1}\left\{\frac{g(x, s)}{\mathcal{K}+s} \int_{0}^{\infty} d \beta e^{-\beta} \exp \left[-\beta \frac{\left(v(\kappa+s)^{-\alpha}+1\right)^{\varsigma} \mathcal{K} k^{2}}{(\kappa+s)^{1-\mu}}\right]\right\} \\
& =\int_{-\infty}^{\infty} d x^{\prime} \frac{g\left(x-x^{\prime}, s\right)}{\kappa+s} \int_{0}^{\infty} d \beta \frac{e^{-\beta}}{\sqrt{\pi}} \frac{(\kappa+s)^{\frac{1-\mu}{2}}}{\sqrt{4\left(v(\kappa+s)^{-\alpha}+1\right)^{\varsigma} \mathcal{K} \beta}} \\
& \times \exp \left[-\frac{(\kappa+s)^{1-\mu}}{4\left(v(\mathcal{K}+s)^{-\alpha}+1\right)^{\varsigma \mathcal{K} \beta}} x^{\prime 2}\right],
\end{aligned}
$$

in which $g(k, s)=1+\kappa \mathcal{F}\{\mathcal{L}\{\delta(x-v t)\}\}$. By doing the integration in $\beta$, we have

$$
\widetilde{p}(x, s)=\int_{-\infty}^{\infty} d x^{\prime} \frac{g\left(x-x^{\prime}, s\right)}{\kappa+s} \frac{(\kappa+s)^{\frac{1-\mu}{2}}}{\sqrt{4\left(v(\kappa+s)^{-\alpha}+1\right)^{\varsigma} \mathcal{K}}} \widetilde{Y}\left(x^{\prime}, s\right),
$$

in which 


$$
\widetilde{Y}(x, s)=\exp \left[-\frac{(\kappa+s)^{\frac{1-\mu}{2}}}{\left(v(\kappa+s)^{-\alpha}+1\right)^{\frac{c}{2}} \sqrt{\mathcal{K}}}|x|\right] .
$$

This equation can be obtained directly from the inverse of Fourier transform of Equation (35); we choose the path presented to bring more detail to the reader. Now, considering

$$
\left|\frac{(\kappa+s)^{\frac{1-\mu}{2}}}{\left(v(\kappa+s)^{-\alpha}+1\right)^{\frac{\zeta}{2}} \sqrt{\mathcal{K}}}\right| x||<1,
$$

at Equation (38) we obtain

$$
\widetilde{Y}(x, s)=\sum_{n=0}^{\infty}(-1)^{n}\left(\frac{(\kappa+s)^{\frac{1-\mu}{2}}}{\left(v(\kappa+s)^{-\alpha}+1\right)^{\frac{\zeta}{2}}}\right)^{n} \frac{|x|^{n}}{\mathcal{K}^{\frac{n}{2}}} .
$$

Performing this inverse Laplace transform not is such an easy task. To facilitate this process, we will rewrite Equation (37) as follows

$$
\begin{aligned}
p(x, t) & =e^{-\kappa t} \mathcal{L}^{-1}\left\{\int_{-\infty}^{\infty} d x^{\prime} \frac{g\left(x-x^{\prime}, s-\kappa\right) s^{-\frac{1+\mu}{2}}}{\sqrt{4\left(v s^{-\alpha}+1\right) \varsigma \mathcal{K}}} \sum_{n=0}^{\infty}(-1)^{n} \frac{|x|^{n}}{\mathcal{K}^{\frac{n}{2}}} \frac{s^{\frac{(1-\mu) n}{2}}}{\left(v s^{-\alpha}+1\right)^{\frac{\zeta n}{2}}}\right\} \\
& =e^{-\kappa t} \mathcal{L}^{-1}\left\{\int_{-\infty}^{\infty} d x^{\prime} \frac{g\left(x-x^{\prime}, s-\kappa\right)}{2 s^{\frac{1+\mu}{2}}} \sum_{n=0}^{\infty}(-1)^{n} \frac{|x|^{n}}{\mathcal{K}^{\frac{n+1}{2}}} \frac{s^{\frac{(1-\mu) n}{2}}}{\left(v s^{-\alpha}+1\right)^{\frac{\varsigma(n+1)}{2}}}\right\} .
\end{aligned}
$$

We need to make the inverse Laplace of this function with $0<\mu<1$. However, it is not possible to use the relation (11) directly. To solve this problem, we use the formula

$$
\mathcal{L}^{-1}\left\{\sqrt{\frac{\pi}{s}} \widetilde{\mathcal{G}}\left[\frac{1}{4 s}\right]\right\}=t^{-\frac{1}{2}} \int_{0}^{\infty} d u \cos [\sqrt{t u}] \mathcal{G}[u],
$$

this formula was introduced by Prudnikov, Brychkov, and Marichev in [79]. So that we can use the formula above, we rewrite Equation (41) as

$$
p(x, t)=e^{-\kappa t} \mathcal{L}^{-1}\left\{\int_{-\infty}^{\infty} d x^{\prime} \frac{g\left(x-x^{\prime}, s-\kappa\right)}{2 s^{\frac{1+\mu}{2}}} \Phi\left(x^{\prime}, s\right)\right\},
$$

in which

$$
\Phi(x, s)=\sum_{n=0}^{\infty}(-1)^{n} \frac{|x|^{n}}{\mathcal{K}^{\frac{n+1}{2}}} \sqrt{\frac{\pi}{s}} \widetilde{\mathcal{G}(s)},
$$

with

$$
\widetilde{\mathcal{G}}(s)=\frac{4^{-\frac{n(1-\mu)+1}{2}}}{\sqrt{\pi}} \frac{\left(\frac{1}{4 s}\right)^{\frac{\alpha \varsigma(n+1)}{2}}}{\left(v 4^{\alpha}\left(\frac{1}{4 s}\right)^{\alpha}+1\right)^{\frac{\zeta(n+1)}{2}}}\left(\frac{1}{\frac{1}{4 s}}\right)^{\frac{(1-\mu) n+1}{2}+\frac{\alpha \zeta(n+1)}{2}},
$$

the $\widetilde{\mathcal{G}}(s)$ function above has the structure of the function $\widetilde{\mathcal{G}}\left(\frac{1}{4 s}\right)$ defined by Formula (42). Using Formula (42), and Equation (11), we can perform the inverse Laplace transform of the function $\Phi$ (Equation (44)), we obtain 


$$
\Phi(x, t)=\int_{0}^{\infty} d u \sum_{n=0}^{\infty} a_{n, \alpha, \mu}^{\varsigma, v} \frac{|x|^{n}}{\mathcal{K}^{\frac{n+1}{2}}} t^{-\frac{1}{2}} \cos [\sqrt{t u}] u^{\frac{\alpha \varsigma(n+1)}{2}+\frac{n(1-\mu)-1}{2}} E_{\alpha, \frac{(n+1) \varsigma}{2}}^{\frac{n(1-\mu)+1}{2}+\frac{\alpha_{\varsigma}(n+1)}{2}}\left[-\frac{u^{\alpha}}{4^{\alpha} v}\right],
$$

in which

$$
a_{n, \alpha, \mu}^{\varsigma, v}=\frac{(-1)^{n}}{\sqrt{\pi} v^{\frac{\zeta(n+1)}{2}} 4^{\frac{\alpha \zeta(n+1)}{2}+\frac{n(1-\mu)+1}{2}}}
$$

using $\Phi(x, t)$ and theorem convolution, we have

$$
p(x, t)=\frac{e^{-\kappa t}}{2} \int_{-\infty}^{\infty} d x^{\prime} \int_{0}^{t} d t^{\prime} g\left(x-x^{\prime}, t-t^{\prime}\right) \int_{0}^{t^{\prime}} d t^{\prime \prime} \frac{t^{\prime \prime} \frac{\mu+1}{2}-1}{\Gamma\left[\frac{\mu+1}{2}\right]} \Phi\left(x^{\prime}, t^{\prime}-t^{\prime \prime}\right)
$$

but $g(x, t)=\delta(t) \delta(x)+\kappa \kappa^{\kappa t} \delta(x-v t)$, therefore

$$
\begin{aligned}
p(x, t) & =\frac{e^{-\kappa t}}{2} \int_{0}^{t} d t^{\prime} \frac{\frac{1}{\prime \frac{\mu+1}{2}}^{2}-1}{\Gamma\left[\frac{\mu+1}{2}\right]} \Phi\left(x, t-t^{\prime}\right) \\
& +\frac{\kappa}{2} \int_{0}^{t} d t^{\prime} \int_{0}^{t^{\prime}} d t^{\prime \prime} \frac{t^{\prime \prime} \frac{\mu+1}{2}-1}{\Gamma\left[\frac{\mu+1}{2}\right]} e^{-\kappa t^{\prime}} \Phi\left(x-v\left(t-t^{\prime}\right), t^{\prime}-t^{\prime \prime}\right) .
\end{aligned}
$$

This solution has several peculiarities. The case without stochastic resetting, i.e., $\kappa=0$ already yields a new result, since the equation of diffusion with the derivative of Prabhakar had been solved only in the Fourier space; see Theorem 5.1 in [51]. When we consider $\kappa>0$, the solution incorporates "stochastic resetting", for the usual static case, i.e., $v=0$, we generalize the Evans-Majumdar model for the case of the Prabhakar derivative. The generalized diffusive process and the solutions have well-defined stationary forms that obey the structure of Equation (14). Finally, for $\kappa>0$ and $v \neq 0$ we have the process of stochastic resetting with non-static resetting as named by us. In this case, the peak of the distribution propagates in space, and at the same time the total probability is conserved. For a more detailed analysis of this and the other cases discussed previously, we will analyze the MSD of these solutions.

\subsection{MSD and Anomalous Diffusion Phenomena}

The MSD is a very important quantity for analyzing the temporal evolution of diffusive and probabilistic systems [19]. The MSD is defined by the following mathematical expression

$$
\left\langle(x-\langle x\rangle)^{2}\right\rangle=\int_{-\infty}^{\infty} d x p(x, t)\left(x-\int_{-\infty}^{+\infty} d x p(x, t) x\right)^{2} .
$$

When a physical system, for example in molecular dynamics, evolves linearly in time, i.e., $\left\langle(x-\langle x\rangle)^{2}\right\rangle \sim t$, the main feature is a random walk in Pearson sense [80]. Hence, if $\left\langle(x-\langle x\rangle)^{2}\right\rangle \sim t^{\rho}$ we have the phenomenon known as fractional dynamics or anomalous diffusion [19]. To recap, anomalous diffusion processes can be classified in the following ways: for $\rho<1$ sub-diffusion, $\rho>1$ over-diffusion, $\rho=1$ ballistic diffusion, and for $\rho>1$ hyper-diffusion.

For systems whose total probability is preserved, i.e., $\int_{-\infty}^{+\infty} p(x, t) d x=1$, we can rewrite the expression (50) as $\left\langle(x-\langle x\rangle)^{2}\right\rangle=\left\langle x^{2}\right\rangle-\langle x\rangle^{2}$. Then, we just must determine $\langle x\rangle$ and $\left\langle x^{2}\right\rangle$ separately. So

$$
\widetilde{\langle x\rangle}(s)=\left.i\left\{\frac{\partial}{\partial k} \widetilde{\bar{p}}(k, s)\right\}\right|_{k=0} \quad \text { and } \quad \widetilde{\left\langle x^{2}\right\rangle}(s)=-\left.\left\{\frac{\partial^{2}}{\partial k^{2}} \widetilde{\bar{p}}(k, s)\right\}\right|_{k=0},
$$


in which the general solution is given by Equation (23), so we have

$$
\widetilde{\langle x\rangle}(s)=\frac{\kappa v}{s^{2}(\kappa+s)},
$$

and

$$
\begin{aligned}
\widetilde{\left\langle x^{2}\right\rangle}(s) & =\frac{2 \mathcal{K}\left(v+(\kappa+s)^{\alpha}\right)^{\varsigma}}{s(\kappa+s)^{1+\alpha \zeta-\mu}}+\frac{2 \kappa v^{2}}{s^{3}(\kappa+s)} \\
& =\frac{2 \mathcal{K}\left(v+(\kappa+s)^{\alpha}\right)^{\varsigma}}{s(\mathcal{K}+s)^{1+\alpha \varsigma^{-\mu}}}+\frac{2 v^{2}}{s^{2}}\left(\frac{1}{s}-\frac{1}{s+\kappa}\right) \\
& =\frac{2 \mathcal{K}\left(v+(\kappa+s)^{\alpha}\right)^{\varsigma}}{s(\kappa+s)^{1+\alpha \varsigma}-\mu}+\frac{2 v^{2}}{s^{3}}-\frac{2 v^{2}}{s \mathcal{K}}\left(\frac{1}{s}-\frac{1}{s+\kappa}\right) \\
& =\frac{2 \mathcal{K}\left(v+(\kappa+s)^{\alpha}\right)^{\varsigma}}{s(\kappa+s)^{1+\alpha-\mu}}+\frac{2 v^{2}}{s^{3}}-\frac{2 v^{2}}{s^{2} \kappa}+\frac{2 v^{2}}{\kappa s(s+\kappa)}
\end{aligned}
$$

the inverse Laplace transform in Equation (52) results in

$$
\langle x\rangle(t)=v \frac{e^{-\kappa t}-1}{\kappa}+t v .
$$

Considering the Formula (11), we can write the exact solution of Equation (53)

$$
\begin{aligned}
\left\langle x^{2}\right\rangle(t) & =2 \mathcal{K} \int_{0}^{t} d t^{\prime} t^{\prime-\mu} e^{-\kappa t^{\prime}} E_{\alpha, 1-\mu}^{-\varsigma}\left(-v t^{\prime \alpha}\right) \\
& +2 v^{2}\left(\frac{t^{2}}{2}-\frac{t}{\mathcal{K}}+\frac{1-e^{-\kappa t}}{\kappa^{2}}\right) .
\end{aligned}
$$

Thus, we can analyze some asymptotic limits. For long times $(t \rightarrow \infty)$, we have the following form

$$
\lim _{t \rightarrow \infty}\left\langle(x-\langle x\rangle)^{2}\right\rangle=2 \mathcal{K} \beta+\frac{v^{2}}{\mathcal{K}^{2}}
$$

with $\beta=2 \frac{\left(\kappa^{\alpha}+v\right)^{\zeta}}{\kappa^{1-\mu+\zeta \alpha}}$ and $\kappa>0$. For this asymptotic limit, $v=0$ we retrieve the expected behavior for the MSD that is constant. Nevertheless, for $v \neq 0$ the resetting point moves, which implies some changes in the known behavior. Figures 2 and 3 show how the velocity of resetting points influences in the system.

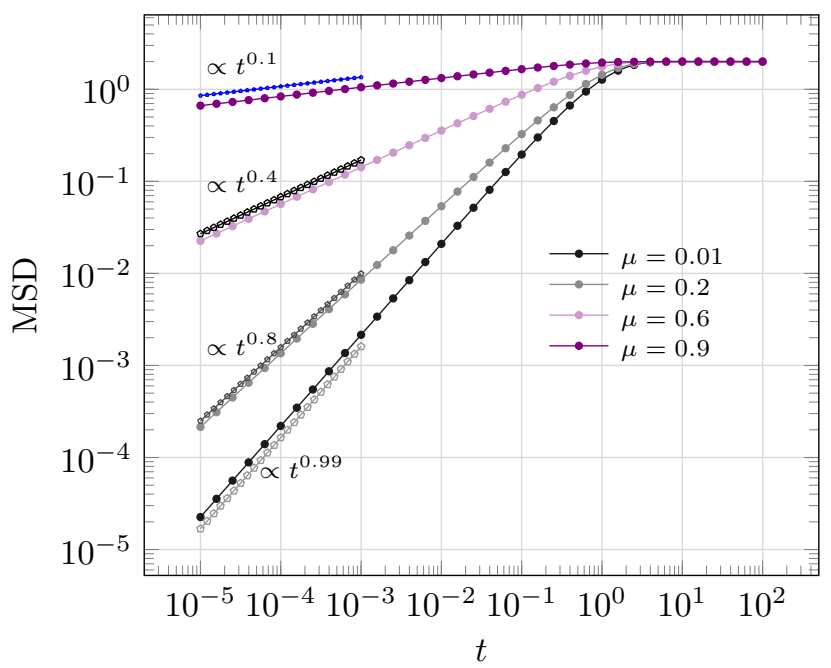

Figure 2. In this figure, we have the MSD to the system with stochastic resetting with zero velocity and with the following values $\kappa=1, \mathcal{K}=1, \varsigma=0$ and $\mu \in\{0.01,0.2,0.6,0.9\}$. 


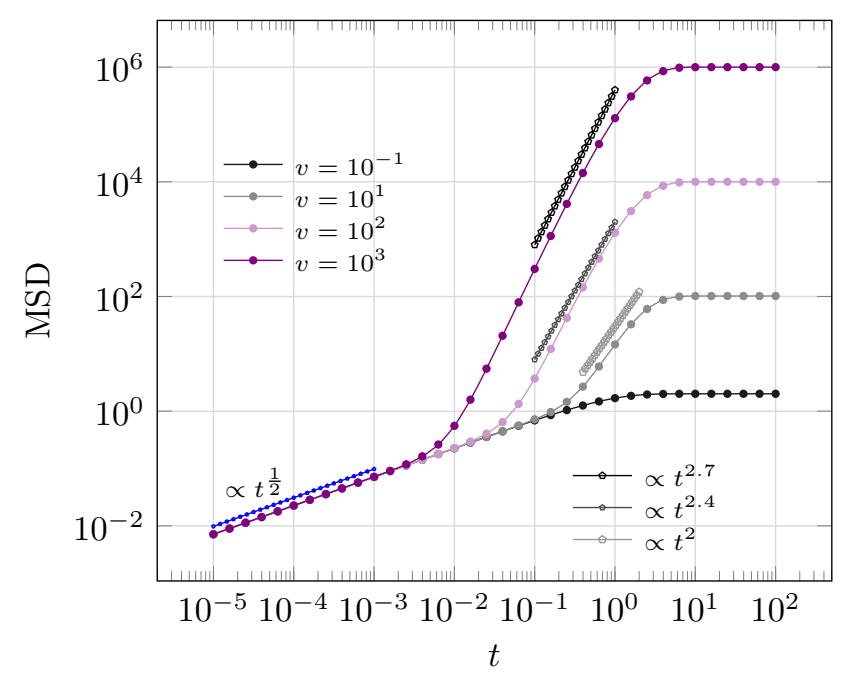

Figure 3. In this figure, we have the MSD for system with stochastic resetting to $v \in$ $\left\{10^{-1}, 10^{1}, 10^{2}, 10^{3}\right\}$, and with the following values $\mathcal{K}=1, \mathcal{K}=1, \varsigma=0$ and $\mu=0.5$.

\section{The Non-Static Stochastic Resetting Theory on Presence of Forces and Multiple Resetting Points}

In the context presented for non-static stochastic resetting theory, we can incorporate the presence of forces in the system, and make an analysis of the dynamics of the system through MSD. Before we start this analysis, it is important to ask the following question: How does the model change if there are more resetting points that move? The answer is simple: we change the variables $v t$ by $v_{n} t$ in Equation (22), and it follows the following form

$$
\begin{aligned}
\mathcal{D}_{\alpha, \mu, t}^{\delta, v, \kappa} p(x, t) & =-\frac{\partial}{\partial x} \mathcal{J}(x, t) \\
& -\int_{0}^{t} d t^{\prime} e^{-\kappa\left(t-t^{\prime}\right)} \wp_{\alpha, \mu}^{\delta, v}\left[t-t^{\prime}\right]\left(\kappa p\left(x, t^{\prime}\right)-\sum_{n} \kappa w_{n} \delta\left(x-v_{n} t^{\prime}\right)\right),
\end{aligned}
$$

in which

$$
\mathcal{J}(x, t)=-\mathcal{K} \frac{\partial}{\partial x} p(x, t)-\left\{\frac{d}{d x} U(x)\right\} p(x, t)
$$

is the probability flux, with $\mathcal{J}( \pm \infty, t)=0$. Here, we have $n$ restart points for the system that is resetting in point $x_{n}=v_{n} t$, in which each has a constant velocity, and may or may not be different. For the system to remain normalized, we consider that Equation (57) obeys the following condition

$$
\sum_{n} w_{n}=1
$$

The term of probability flux (58) includes the presence of forces, let us consider the following potential

$$
U(x)=b_{0}+b_{1} x+b_{2} x^{2} .
$$

Now, we can go on with our idea of calculating MSD, which consists of determining the quantities $\langle x\rangle$ and $\left\langle x^{2}\right\rangle$. Therefore, by multiplying Equation (57) by $x^{2}$ and then performing the integration in $x$-variable, we have 


$$
\begin{aligned}
\mathcal{D}_{\alpha, \mu, t}^{\delta, v, \kappa}\left\langle x^{2}\right\rangle & =2 \int_{-\infty}^{+\infty} d x x \mathcal{J}(x, t) \\
& -\int_{0}^{t} d t^{\prime} e^{-\kappa\left(t-t^{\prime}\right)} \wp_{\alpha^{\prime}, \mu}^{\delta, v}\left(t-t^{\prime}\right)\left(\kappa\left\langle x^{2}\right\rangle+\kappa \sum_{n} w_{n} v_{n}^{2} t^{2}\right),
\end{aligned}
$$

the integral of the $x \mathcal{J}(x, t)$ for a quadratic potential is given by

$$
\begin{aligned}
\int_{-\infty}^{+\infty} d x x \mathcal{J}(x, t) & =\mathcal{K}+\int_{-\infty}^{+\infty} d x x\left(-b_{1}-b_{2} x\right) p(x, t) \\
& =\mathcal{K}-b_{1}\langle x\rangle-b_{2}\left\langle x^{2}\right\rangle
\end{aligned}
$$

replacing this equation in Equation (61) and realizing the Laplace transform, we obtain

$$
\begin{aligned}
\frac{(\kappa+s)^{\alpha \delta-\mu_{s}} \widetilde{\left.v^{2}\right\rangle}}{\left(v+\left((\kappa+s)^{\alpha}\right)^{\delta}\right.} & =\frac{2 \mathcal{K}}{s}-2 b_{1} \widetilde{\langle x\rangle}-2 b_{2} \widetilde{\left\langle x^{2}\right\rangle} \\
& -\frac{(\kappa+s)^{\alpha \delta-\mu}}{\left(v+\left((\kappa+s)^{\alpha}\right)^{\delta}\right.} \kappa \widetilde{\left\langle x^{2}\right\rangle} \\
& -\frac{(\kappa+s)^{\alpha \delta-\mu}}{\left(v+\left((\kappa+s)^{\alpha}\right)^{\delta}\right.} \sum_{n} w_{n} \mathcal{L}\left\{\kappa v_{n}^{2} t^{2}\right\},
\end{aligned}
$$

which implies

$$
\begin{aligned}
\widetilde{\left\langle x^{2}\right\rangle}(s) & =\Psi_{1}(s)\left(\frac{2 \mathcal{K}}{s}-2 b_{1} \widetilde{\langle x\rangle}\right) \\
& +\Psi_{2}(s) \sum_{n} w_{n} \mathcal{L}\left\{\kappa v_{n}^{2} t^{2}\right\},
\end{aligned}
$$

in which

$$
\Psi_{1}(s)=\frac{\left(v+\left((\kappa+s)^{\alpha}\right)^{\delta}\right.}{(\kappa+s)^{1+\alpha \delta-\mu}+2 b_{2}\left(v+\left((\kappa+s)^{\alpha}\right)^{\delta}\right.}
$$

and

$$
\Psi_{2}(s)=\frac{(\kappa+s)^{\alpha \delta-\mu}}{(\kappa+s)^{1+\alpha \delta-\mu}+2 b_{2}\left(v+\left((\kappa+s)^{\alpha}\right)^{\delta}\right.} .
$$

Now we need to determine the quantity $\widetilde{\langle x\rangle}$, for this we simply multiply Equation (57) by $x$ and integrate in the same variable $x$, we obtain

$$
\begin{aligned}
\frac{(\kappa+s)^{\alpha \delta-\mu_{s}}}{\left(v+\left((\kappa+s)^{\alpha}\right)^{\delta}\right.} \widetilde{\langle x\rangle}(s) & =-\frac{b_{1}}{s}-b_{2} \widetilde{\langle x\rangle} \\
& -\frac{(\kappa+s)^{\alpha \delta-\mu}}{\left(v-\left((\kappa+s)^{\alpha}\right)^{\delta}\right.}\left(\kappa \widetilde{\langle x\rangle}+\sum_{n} w_{n} \mathcal{L}\left\{\kappa v_{n} t\right\}\right),
\end{aligned}
$$

this equation can be rewritten as

$$
\widetilde{\langle x\rangle}(s)=\frac{1}{\frac{(\kappa+s)^{1-\mu}}{\left(1+v(\kappa+s)^{-\alpha}\right)^{\delta}}+b_{2}}\left(-\frac{b_{1}}{s}+\frac{\sum_{n} w_{n} \mathcal{L}\left\{\kappa v_{n} t\right\}}{(\kappa+s)^{\mu-\alpha \delta}\left(v+\left((\kappa+s)^{\alpha}\right)^{\delta}\right.}\right),
$$


performing a power series expansion of the type $(1-x)^{-1}=\sum_{i=0}^{\infty} x^{i}$, which implies

$$
\widetilde{\langle x\rangle}(s)=-\sum_{i=0}^{\infty}(-1)^{i} \widetilde{\gamma}_{1}(s) b_{2}^{i} \frac{b_{1}}{s}+\sum_{i=0}^{\infty}(-1)^{i} \widetilde{\gamma}_{2}(s) b_{2}^{i} \sum_{n} w_{n} \mathcal{L}\left\{\kappa v_{n} t\right\},
$$

with the following coefficients

$$
\begin{aligned}
& \widetilde{\gamma}_{1}(s)=(\kappa+s)^{-(1-\mu)(i+1)}\left(1+v(\kappa+s)^{-\alpha}\right)^{(i+1) \delta}, \\
& \widetilde{\gamma}_{2}(s)=(\kappa+s)^{-(1-\mu) i-1}\left(1+v(\kappa+s)^{-\alpha}\right)^{i \delta} .
\end{aligned}
$$

Therefore, the quantity $\widetilde{\langle x\rangle}(s)$ has the following inverse Laplace transform

$$
\begin{aligned}
\langle x\rangle(t) & =-\sum_{i=0}^{\infty}(-1)^{i} \int_{0}^{t^{\prime}} d t^{\prime} \gamma_{1}\left(t^{\prime}\right) b_{2}^{i} b_{1} \\
& +\sum_{i=0}^{\infty} \sum_{n} w_{n}(-1)^{i} b_{2}^{i} \int_{0}^{t} d t^{\prime} \gamma_{2}\left(t-t^{\prime}\right) \kappa v_{n} t^{\prime},
\end{aligned}
$$

in which $\gamma_{1}(t)$ e $\gamma_{2}(t)$ can be determined by the Formula (11), the results are

$$
\begin{aligned}
& \gamma_{1}(t)=e^{-\kappa t} t^{(1-\mu)(i+1)-1} E_{\alpha,(1-\mu)(i+1)}^{-(i+1) \delta}\left(-v t^{\alpha}\right), \\
& \gamma_{2}(t)=e^{-\kappa t} t^{(1-\mu) i} E_{\alpha,(1-\mu) i+1}^{-(i+1) \delta+1}\left(-v t^{\alpha}\right) .
\end{aligned}
$$

Thus, we can use Equation (65) and write the inverse transform as follows

$$
\begin{aligned}
\left\langle x^{2}\right\rangle(t) & =\int_{0}^{t} d t^{\prime} \Psi_{1}\left(t^{\prime}\right)\left(2 \mathcal{K}-2 b_{1}\langle x\rangle\left(t-t^{\prime}\right)\right) \\
& +\mathcal{L}^{-1}\left\{\Psi_{2}(s) \sum_{n} w_{n} \mathcal{L}\left\{\kappa v_{n}^{2} t^{2}\right\}\right\}
\end{aligned}
$$

in which the function $\Psi_{i}$ is defined in Equations (66) and (67). We can rewrite the $\Psi_{1,2}(s)$ functions in power series

$$
\Psi_{1,2}(s)=\sum_{i=0}^{\infty}(-1)^{i} \widetilde{\gamma}_{1,2}(s) 2^{i-1} b_{2}^{i}
$$

in which the inverse transform of $\widetilde{\gamma}_{1,2}(s)$ is given by Equations (74) and (75). Thus, we have the following expressed for Equation (76)

$$
\begin{aligned}
\left\langle x^{2}\right\rangle(t) & =\int_{0}^{t} d t^{\prime} \Psi_{1}\left(t-t^{\prime}\right)\left(2 \mathcal{K}-2 b_{1}\langle x\rangle\left(t^{\prime}\right)\right) \\
& +\sum_{n}^{\infty} w_{n} \int_{0}^{t} d t^{\prime} \Psi_{2}\left(t-t^{\prime}\right) \kappa v_{n}^{2} t^{\prime 2}
\end{aligned}
$$

in which

$$
\begin{aligned}
& \Psi_{1}(t)=\sum_{i=0}^{\infty}(-1)^{i} \gamma_{1}(t) 2^{i-1} b_{2}^{i}, \\
& \Psi_{2}(t)=\sum_{i=0}^{\infty}(-1)^{i} \gamma_{2}(t) 2^{i-1} b_{2}^{i},
\end{aligned}
$$

the $\gamma_{1,2}$-functions was defined in Equations (74) and (75). These exact expressions for the moments $\left(\left\langle x^{i}\right\rangle\right.$ with $\left.i \in\{1,2\}\right)$ reveal a strong dependence on the variable resetting point in the diffusion of 
these walkers, partly because of the presence of external forces that we add to the system through the probability flux $\mathcal{J}(p(x, t))$. This problem of multiple resetting points has an extreme degree of complexity, but in this section we show the exact expressions to $\langle x\rangle$ and $\left\langle x^{2}\right\rangle$ that allow us to write the MSD, i.e., $\left\langle x^{2}\right\rangle-\langle x\rangle^{2}$. This mathematical-physics application reveals the great potential that the stochastic resetting model has in complex systems.

\section{Conclusions}

In this work we have investigated a non-static stochastic resetting theory to generalized FPE with Prabhakar fractional operator. Using the Laplace transform, we have obtained general analytical solutions of the fractional FPE in a more general context, i.e., tempered $\wp_{\alpha, \beta}^{\delta, v}(t)$-Prabhakar kernel.

The model proposed generalizes the stochastic resetting process (in an Evans-Majumdar sense) to the non-static position of restart. In other words, we consider a non-fixed resetting point. In addition, we take into account the fractional Prabhakar operator. We presented the exact analytic solutions to non-static stochastic resetting theory for three cases

- Usual diffusion;

- Fractional Caputo diffusion;

- Fractional Prabhakar diffusion;

in addition to finding the exact solutions, we show how these solutions connect with the renewal process. Through MSD to this model we show that the system presents anomalous diffusive regimes. We show that considering the fractional case and the $v \neq 0$ ( $v$ is velocity) of the resetting position the MSD has an initial sub-diffusive regime and then hyper-diffusive regime, before the MSD has acquired a stationary form, i.e., MSD constant. Finally, we generalize the model to the case where it admits multiple reset points that move with different velocities. In addition, we consider the presence of external forces acting in the system, assuming the form $F(x)=-b_{1}-b_{2} x$. We found the exact expressions for the first moment and second moment.

We consider the results and techniques employed in this work to constitute important tools for studying non-Markovian diffusive process with memory effects, thus opening new possibilities in future research for fractional diffusion and stochastic resetting theory.

Funding: This research received no external funding.

Acknowledgments: This work was partially supported by Brazilian agency CNPq. We thank the referees for their comments and recommendations.

Conflicts of Interest: The authors declare no conflict of interest.

\section{References}

1. Klages, R. Search for food of birds, fish and insects. In Diffusive Spreading in Nature, Technology and Society; Springer: Berlin, Germany, 2018; pp. 49-69.

2. Noetel, J.; Freitas, V.; Macau, E.; Schimansky-Geier, L. Optimal noise in a stochastic model for local search. Phys. Rev. E 2018, 98, 022128. [CrossRef] [PubMed]

3. Reuveni, S. Optimal stochastic restart renders fluctuations in first passage times universal. Phys. Rev. Lett. 2016, 116, 170601. [CrossRef] [PubMed]

4. Pal, A.; Kundu, A.; Evans, M.R. Diffusion under time-dependent resetting. J. Phys. A Math. Theor. 2016, 49, 225001. [CrossRef]

5. Belan, S. Restart could optimize the probability of success in a Bernoulli trial. Phys. Rev. Lett. 2018, 120, 080601. [CrossRef] [PubMed]

6. Berg, O.G.; Winter, R.B.; Von Hippel, P.H. Diffusion-driven mechanisms of protein translocation on nucleic acids. 1. Models and theory. Biochemistry 1981, 20, 6929-6948. [CrossRef] [PubMed]

7. Coppey, M.; Bénichou, O.; Voituriez, R.; Moreau, M. Kinetics of target site localization of a protein on DNA: A stochastic approach. Biophys. J. 2004, 87, 1640-1649. [CrossRef] [PubMed] 
8. Lomholt, M.A.; van den Broek, B.; Kalisch, S.M.J.; Wuite, G.J.; Metzler, R. Facilitated diffusion with DNA coiling. Proc. Natl. Acad. Sci. USA 2009, 106, 8204-8208. [CrossRef] [PubMed]

9. Evans, M.R.; Majumdar, S.N. Run and tumble particle under resetting: A renewal approach. J. Phys. A Math. Theor. 2018, 51, 475003. [CrossRef]

10. Evans, M.R.; Majumdar, S.N. Diffusion with stochastic resetting. Phys. Rev. Lett. 2011, $106,160601$. [CrossRef] [PubMed]

11. Majumdar, S.N.; Sabhapandit, S.; Schehr, G. Dynamical transition in the temporal relaxation of stochastic processes under resetting. Phys. Rev. E 2015, 91, 052131. [CrossRef] [PubMed]

12. Durang, X.; Henkel, M.; Park, H. The statistical mechanics of the coagulation-diffusion process with a stochastic reset. J. Phys. A Math. Theor. 2014, 47, 045002. [CrossRef]

13. Eule, S.; Metzger, J.J. Non-equilibrium steady states of stochastic processes with intermittent resetting. New J. Phys. 2016, 18, 033006. [CrossRef]

14. Roldán, É.; Lisica, A.; Sánchez-Taltavull, D.; Grill, S.W. Stochastic resetting in backtrack recovery by RNA polymerases. Phys. Rev. E 2016, 93, 062411. [CrossRef] [PubMed]

15. Risken, H. Fokker-Planck Equation; Springer: Berlin, Germany, 1996; pp. 63-95.

16. Frank, T.D. Nonlinear Fokker-Planck Equations: Fundamentals and Applications; Springer Science \& Business Media: Berlin, Germany, 2005.

17. Tarasov, V.E. Fractional hydrodynamic equations for fractal media. Ann. Phys. 2005, 318, 286-307. [CrossRef]

18. Metzler, R.; Barkai, E.; Klafter, J. Deriving fractional Fokker-Planck equations from a generalised master equation. EPL Europhys. Lett. 1999, 46, 431. [CrossRef]

19. Metzler, R.; Barkai, E.; Klafter, J. Anomalous diffusion and relaxation close to thermal equilibrium: A fractional Fokker-Planck equation approach. Phys. Rev. Lett. 1999, 82, 3563. [CrossRef]

20. Lua, R.C.; Grosberg, A.Y. First passage times and asymmetry of DNA translocation. Phys. Rev. E 2005, 72, 061918. [CrossRef] [PubMed]

21. Dieterich, P.; Klages, R.; Preuss, R.; Schwab, A. Anomalous dynamics of cell migration. Proc. Natl. Acad. Sci. USA 2008, 105, 459-463. [CrossRef] [PubMed]

22. Lomholt, M.A.; Ambjörnsson, T.; Metzler, R. Optimal target search on a fast-folding polymer chain with volume exchange. Phys. Rev. Lett. 2005, 95, 260603. [CrossRef] [PubMed]

23. Sokolov, I.M. Models of anomalous diffusion in crowded environments. Soft Matter 2012, 8, $9043-9052$. [CrossRef]

24. Barkai, E.; Metzler, R.; Klafter, J. From continuous time random walks to the fractional Fokker-Planck equation. Phys. Rev. E 2000, 61, 132. [CrossRef]

25. Barkai, E. Fractional Fokker-Planck equation, solution, and application. Phys. Rev. E 2001, 63, 046118. [CrossRef] [PubMed]

26. del Castillo-Negrete, D. Fractional diffusion models of nonlocal transport. Phys. Plasmas 2006, 13, 082308. [CrossRef]

27. Hristov, J. Diffusion models with weakly singular kernels in the fading memories. Therm. Sci. 2015, 19, 947-957. [CrossRef]

28. Metzler, R.; Klafter, J. The random walk's guide to anomalous diffusion: A fractional dynamics approach. Phys. Rep. 2000, 339, 1-77. [CrossRef]

29. dos Santos, M. Non-Gaussian Distributions to Random Walk in the Context of Memory Kernels. Fractal Fract. 2018, 2, 20. [CrossRef]

30. Tomovski, Ž.; Sandev, T.; Metzler, R.; Dubbeldam, J. Generalized space-time fractional diffusion equation with composite fractional time derivative. Phys. A Stat. Mech. Appl. 2012, 391, 2527-2542. [CrossRef]

31. Deng, W.; Barkai, E. Ergodic properties of fractional Brownian-Langevin motion. Phys. Rev. E 2009, 79, 011112. [CrossRef] [PubMed]

32. Jeon, J.H.; Tejedor, V.; Burov, S.; Barkai, E.; Selhuber-Unkel, C.; Berg-Sørensen, K.; Oddershede, L.; Metzler, R. In vivo anomalous diffusion and weak ergodicity breaking of lipid granules. Phys. Rev. Lett. 2011, 106, 048103. [CrossRef] [PubMed]

33. Richardson, L.F. Atmospheric diffusion shown on a distance-neighbour graph. Proc. R. Soc. Lond. A 1926, 110, 709-737. [CrossRef]

34. Scher, H.; Montroll, E.W. Anomalous transit-time dispersion in amorphous solids. Phys. Rev. B 1975, $12,2455$. [CrossRef] 
35. Atangana, A.; Koca, I. Chaos in a simple nonlinear system with Atangana-Baleanu derivatives with fractional order. Chaos Solitons Fractals 2016, 89, 447-454. [CrossRef]

36. dos Santos, M.A.F.; Gomez, I.S. A fractional Fokker-Planck equation for non-singular kernel operators. J. Stat. Mech. Theory Exp. 2018, 2018, 123205. [CrossRef]

37. Longhi, S. Fractional Schrödinger equation in optics. Opt. Lett. 2015, 40, 1117-1120. [CrossRef] [PubMed]

38. Giusti, A.; Colombaro, I. Prabhakar-like fractional viscoelasticity. Commun. Nonlinear Sci. Numer. Simul. 2018, 56, 138-143. [CrossRef]

39. Sabzikar, F.; Meerschaert, M.M.; Chen, J. Tempered fractional calculus. J. Comput. Phys. 2015, 293, 14-28. [CrossRef] [PubMed]

40. Prabhakar, T.R. A singular integral equation with a generalized Mittag Leffler function in the kernel. Yokohama Math. J. 1971, 19, 7-15.

41. Loverro, A. Fractional Calculus: History, Definitions and Applications for the Engineer; Rapport Technique; Univeristy of Notre Dame, Department of Aerospace and Mechanical Engineering: Notre Dame, IN, USA, 2004; pp. 1-28.

42. Ross, B. The development of fractional calculus 1695-1900. Hist. Math. 1977, 4, 75-89. [CrossRef]

43. Podlubny, I. Fractional Differential Equations: An Introduction to Fractional Derivatives, Fractional Differential Equations, to Methods of Their Solution and Some of Their Applications; Elsevier: Amsterdam, The Netherlands, 1998; Volume 198.

44. Gorenflo, R.; Kilbas, A. Mittag-Leffler Functions, Related Topics and Applications; Springer: Berlin, Germany, 2014.

45. Bouchaud, J.P.; Georges, A. Anomalous diffusion in disordered media: Statistical mechanisms, models and physical applications. Phys. Rep. 1990, 195, 127-293. [CrossRef]

46. Laskin, N. Fractional schrödinger equation. Phys. Rev. E 2002, 66, 056108. [CrossRef] [PubMed]

47. Wu, X.; Deng, W.; Barkai, E. Tempered fractional Feynman-Kac equation: Theory and examples. Phys. Rev. E 2016, 93, 032151. [CrossRef] [PubMed]

48. Meerschaert, M.M.; Sabzikar, F. Tempered fractional Brownian motion. Stat. Probab. Lett. 2013, 83, $2269-2275$. [CrossRef]

49. Li, C.; Deng, W. High order schemes for the tempered fractional diffusion equations. Adv. Comput. Math. 2016, 42, 543-572. [CrossRef]

50. Kilbas, A.A.; Saigo, M.; Saxena, R.K. Generalized Mittag-Leffler function and generalized fractional calculus operators. Integr. Transform. Spec. Funct. 2004, 15, 31-49. [CrossRef]

51. Garra, R.; Gorenflo, R.; Polito, F.; Tomovski, Ž. Hilfer-Prabhakar derivatives and some applications. Appl. Math. Comput. 2014, 242, 576-589. [CrossRef]

52. Colombaro, I.; Giusti, A.; Vitali, S. Storage and dissipation of energy in Prabhakar viscoelasticity. Mathematics 2018, 6, 15. [CrossRef]

53. Liang, X.; Yang, Y.G.; Gao, F.; Yang, X.J.; Xue, Y. Anomalous Advection-Dispersion Equations within General Fractional-Order Derivatives: Models and Series Solutions. Entropy 2018, 20, 78. [CrossRef]

54. Sandev, T.; Deng, W.; Xu, P. Models for characterizing the transition among anomalous diffusions with different diffusion exponents. J. Phys. A Math. Theor. 2018, 51, 405002. [CrossRef]

55. Stanislavsky, A.; Weron, A. Transient anomalous diffusion with Prabhakar-type memory. J. Chem. Phys. 2018, 149, 044107. [CrossRef] [PubMed]

56. Liemert, A.; Sandev, T.; Kantz, H. Generalized Langevin equation with tempered memory kernel. Phys. A Stat. Mech. Appl. 2017, 466, 356-369. [CrossRef]

57. Sandev, T. Generalized Langevin equation and the Prabhakar derivative. Mathematics 2017, 5, 66. [CrossRef]

58. Fernandez, A.; Baleanu, D.; Srivastava, H. Series representations for fractional-calculus operators involving generalised Mittag-Leffler functions. Commun. Nonlinear Sci. Numer. Simul. 2019, 67, 517-527. [CrossRef]

59. Agarwal, P.; Al-Mdallal, Q.; Cho, Y.J.; Jain, S. Fractional differential equations for the generalized Mittag-Leffler function. Adv. Differ. Equ. 2018, 2018, 58. [CrossRef]

60. Atangana, A.; Baleanu, D. New fractional derivatives with non-local and non-singular kernel: Theory and Application to Heat Transfer Model. arXiv 2016, arXiv:1602.03408

61. Koca, I. Modelling the spread of Ebola virus with Atangana-Baleanu fractional operators. Eur. Phys. J. Plus 2018, 133, 100. [CrossRef] 
62. Avc1, D.; Yetim, A. Analytical solutions to the advection-diffusion equation with the Atangana-Baleanu derivative over a finite domain. Balıkesir Üniversitesi Fen Bilimleri Enstitüsü Dergisi 2018, 20, 382-395. [CrossRef]

63. Majumdar, S.N.; Oshanin, G. Spectral content of fractional Brownian motion with stochastic reset. J. Phys. A Math. Theor. 2018, 51, 435001. [CrossRef]

64. Boyer, D.; Evans, M.R.; Majumdar, S.N. Long time scaling behaviour for diffusion with resetting and memory. J. Stat. Mech. Theory Exp. 2017, 2017, 023208. [CrossRef]

65. Evans, M.R.; Majumdar, S.N.; Mallick, K. Optimal diffusive search: Nonequilibrium resetting versus equilibrium dynamics. J. Phys. A Math. Theor. 2013, 46, 185001. [CrossRef]

66. Pal, A.; Reuveni, S. First passage under restart. Phys. Rev. Lett. 2017, 118, 030603. [CrossRef] [PubMed]

67. Masoliver, J. Telegraphic processes with stochastic resetting. Phys. Rev. E 2019, 99, 012121. [CrossRef] [PubMed]

68. Villarroel, J.; Montero, M. Continuous-time ballistic process with random resets. J. Stat. Mech. Theory Exp. 2018, 2018, 123204. [CrossRef]

69. Pal, A.; Rahav, S. Integral fluctuation theorems for stochastic resetting systems. Phys. Rev. E 2017, 96, 062135. [CrossRef] [PubMed]

70. Fuchs, J.; Goldt, S.; Seifert, U. Stochastic thermodynamics of resetting. EPL (Europhys. Lett.) 2016, 113, 60009. [CrossRef]

71. Henry, B.I.; Wearne, S.L. Fractional reaction-diffusion. Phys. A Stat. Mech. Appl. 2000, 276, 448-455. [CrossRef]

72. Rebenshtok, A.; Barkai, E. Weakly non-ergodic statistical physics. J. Stat. Phys. 2008, 133, 565-586. [CrossRef]

73. Thiel, F.; Sokolov, I.M. Weak ergodicity breaking in an anomalous diffusion process of mixed origins. Phys. Rev. E 2014, 89, 012136. [CrossRef] [PubMed]

74. Barkai, E.; Garini, Y.; Metzler, R. Strange kinetics of single molecules in living cells. Phys. Today 2012, 65, 29. [CrossRef]

75. Metzler, R.; Schick, W.; Kilian, H.G.; Nonnenmacher, T.F. Relaxation in filled polymers: A fractional calculus approach. J. Chem. Phys. 1995, 103, 7180-7186. [CrossRef]

76. Garra, R.; Garrappa, R. The Prabhakar or three parameter Mittag-Leffler function: Theory and application. Commun. Nonlinear Sci. Numer. Simul. 2018, 56, 314-329. [CrossRef]

77. Giusti, A. A comment on some new definitions of fractional derivative. Nonlinear Dyn. 2018, 93, 1757-1763. [CrossRef]

78. Mainardi, F.; Pagnini, G.; Saxena, R. Fox H functions in fractional diffusion. J. Comput. Appl. Math. 2005, 178, 321-331. [CrossRef]

79. Prudnikov, A.P.; Brychkov, Y.A.; Marichev, O.I. Volume 4: Direct Laplace Transforms. Volume 5: Inverse Laplace Transforms. In Integrals and Series; CRC Press: Boca Raton, FL, USA, 1992.

80. Pearson, K. The problem of the random walk. Nature 1905, 72, 342. [CrossRef]

(C) 2019 by the authors. Licensee MDPI, Basel, Switzerland. This article is an open access article distributed under the terms and conditions of the Creative Commons Attribution (CC BY) license (http://creativecommons.org/licenses/by/4.0/). 